Our thanks to all those who have helped with this issue of Bioanalysis. Listed below are authors, referees and others who have kindly given their time, effort and expertise; their generosity has helped establish this publication.

$\begin{array}{llll}\text { Amaravadi L } & \text { Gao Q } & \text { Lin S } & \text { Sun L } \\ \text { Angelillo-Scherrer A } & \text { Gleason C } & \text { Lin T } & \text { Tissot J } \\ \text { Bautista A } & \text { Griffith W } & \text { Lindsay J } & \text { Verch T } \\ \text { Bennett P } & \text { Guillarme D } & \text { Liu G } & \text { Waldvogel M } \\ \text { Beyerlein D } & \text { Henion J } & \text { Lund E } & \text { Wang T } \\ \text { Bouaraphan S } & \text { Hollegaard M } & \text { Ma Y } & \text { Wang Y } \\ \text { Bromirski M } & \text { Huang H } & \text { McKenzie D } & \text { Weng N } \\ \text { Cancilla M } & \text { Huang X } & \text { McMullen J } & \text { White J } \\ \text { Castagna A } & \text { Hucker R } & \text { Needham S } & \text { Wiseman J } \\ \text { Chace D } & \text { Jawa V } & \text { O'Dwyer B } & \text { Woolf E } \\ \text { Chamberlain P } & \text { Jiang Z } & \text { Oliveira R } & \text { Xiao Y } \\ \text { Christianson C } & \text { Johnson C } & \text { Patrone L } & \text { Xie S } \\ \text { Corso G } & \text { Kasiotis K } & \text { Peduzzi D } & \text { Yarovoi H } \\ \text { deJesus V } & \text { Kissinger P } & \text { Premkumar N } & \text { Ye M } \\ \text { Ding J } & \text { Konrad R } & \text { Retzlaff M } & \text { Zhang L } \\ \text { Favrat B } & \text { Koren E } & \text { Rochat B } & \text { Zhang T } \\ \text { Favre A } & \text { Kostense S } & \text { Samanidou V } & \text { Zhou L } \\ \text { Fillet M } & \text { Kottelat E } & \text { Shih S } & \text { Zhou W } \\ \text { Frey T } & \text { Kvaskoff D } & \text { Sikanen T } & \text { Zimmer D } \\ \text { Fuh M } & \text { Lee M } ~ & \text { Snee R } & \text { Zimmer J } \\ \text { Fung E } & \text { Liebisch G } & \text { Staub C } & \text { Zulkoski J } \\ & & & \end{array}$

\title{
INDEX OF ADVERTISERS
}

\section{Company}

Bioanalysis Zone - get in the zone

Join the Bioanalysis Zone

Publish your research with Bioanalysis

Bioanalysis trial

Advertise with Bioanalysis

Subscribe to Bioanalysis 